\title{
Pressure Ulcer Associated with Critical Colonization Successfully Treated by Transient Usage of Cadexomer-Iodine: A Case Report
}

\author{
Shigeki Inui ${ }^{*}, 1$, Toshiko Harada ${ }^{2}$ and Satoshi Itami $^{1}$ \\ ${ }^{I}$ Departments of Regenerative Dermatology Osaka University Graduate School of Medicine and ${ }^{2}$ Takarazuka University \\ School of Nursing, Japan
}

\begin{abstract}
A 56-year-old Japanese man hospitalized for schizophrenia and depression developed pressure ulcer on his greater trochanter due to a long-term bed rest. In spite of applying $0.003 \%$ alprostadil alfadex ointment for two months, the ulcer was not improved. Because there was some purulent discharge, we examined semi-quantitative swab bacterial culture from the ulcer and subsequently detected quadrant III of bacteria. Then, $0.9 \%$ cadexomer-iodine ointment was applied once a day, resulting in decrease of the discharge and only quadrant I of bacteria culture. Thereafter, by application of polyurethane foams for two months, the wound was completely epithelized. The remarkable acceleration of wound healing after using cadexomer-iodine ointment suggested the initial critical colonization, which might have caused delayed healing.
\end{abstract}

Keywords: Pressure ulcer, critical colonization, cadexomer-iodine ointment.

\section{INTRODUCTION}

Chronic wounds are mostly contaminated with bacteria without harmful effect on healing process. However, when bacteria proliferate up to critical threshold of $10^{5}$ bacteria per gram of tissue, increased toxins and inflammatory mediators may cause local tissue damage and consequently delay of wound healing [1]. This condition is referred as critical colonization. The prevalence of pressure ulcers among nursing home residents was reported to be $7 \%$ to $23 \%$ and the incidence of pressure ulcers is estimated to be $14 / 1000$ patient-days among high-risk patients [2]. Further, one prospective study of 16 patients with pressure ulcers who were followed for 2184 days reported that the incidence of infection was 1.4 cases per 1000 patient-ulcer days [3]. However, the prevalence of critical colonization in pressure ulcers has not been reported possibly because the differential diagnosis is difficult between critical colonization and infection. Here, we report a case of pressure ulcer associated with critical colonization successfully treated by transient usage of cadexomer-iodine.

\section{CASE PRESENTATION}

A 56-year-old Japanese man hospitalized for schizophrenia and depression developed pressure ulcer on his greater trochanter due to a long-term bed rest. In spite of applying $0.003 \%$ alprostadil alfadex ointment (Prostandin ${ }^{\circledR}$ ointment $0.003 \%$, Ono pharmaceutical Co. Ltd., Osaka, Japan) for two months, the ulcer was not improved (Fig. 1A). Because there was some purulent discharge, we examined semi-quantitative swab bacterial culture from the

*Address correspondence to this author at the Departments of Regenerative Dermatology Osaka University Graduate School of Medicine, 2-2, G2, Yamadaoka, Suita-shi, Osaka 5650871, Japan; Tel: +81 66879 3960;

Fax: +81 66879 6962; E-mail: inui@ @-derma.med.osaka-u.ac.jp ulcer. Briefly, the bacterial swabs were inoculated onto standard media in a Petri dish and serially diluted and streaked into four quadrants. Five days later, bacterial species isolated from the four quadrants were evaluated as scant (I, first quadrant), light (II, second quadrant), moderate (III, third quadrant), or heavy growth (IV, fourth quadrant). Then we detected quadrant III of Streptococcus agalactiae and Bacteroides fragilis, quadrant II of $\alpha$-Streptococcus, and quadrant I of methicillin-sensitive Staphylococcus aureus (MSSA) and Neisseria species. Although there were no signs of infection but the purulent discharge, $0.9 \%$ cadexomeriodine ointment was applied once a day for a week, resulting in decrease of the discharge (Fig. 1B). Simultaneous semiquantitative swab bacterial culture showed only quadrant I of MSSA and Streptococcus agalactiae. Then, the cadexomeriodine ointment was withdrawn and polyurethane foams (HydroSite ${ }^{\circledR}$, Smith \& Nephew, London, UK) were applied. One month later the wound size gradually decreased (Fig. 1C) and one more month later the wound was completely epithelized (Fig. 1D). The remarkable acceleration of wound healing after using cadexomer-iodine ointment suggested the initial critical colonization, which might have caused delayed healing.

\section{COMMENTS}

Very recently, a standardized UPPER and LOWER mnemonic for wound infection checklist was developed to diagnose critical colonization and deep infection in a randomized controlled trial to evaluate an antimicrobial dressing with silver alginate powder [4]. The UPPER, which is associated with critical colonization, refers to the symptoms of unhealthy tissue, pain, poor healing, exudate and reek and at least 2 signs are required for the identification of critical colonization. On the other hand, the LOWER, associated with deep infection, refers to larger in size, osseous tissue, warmth, edema and redness. In our case 


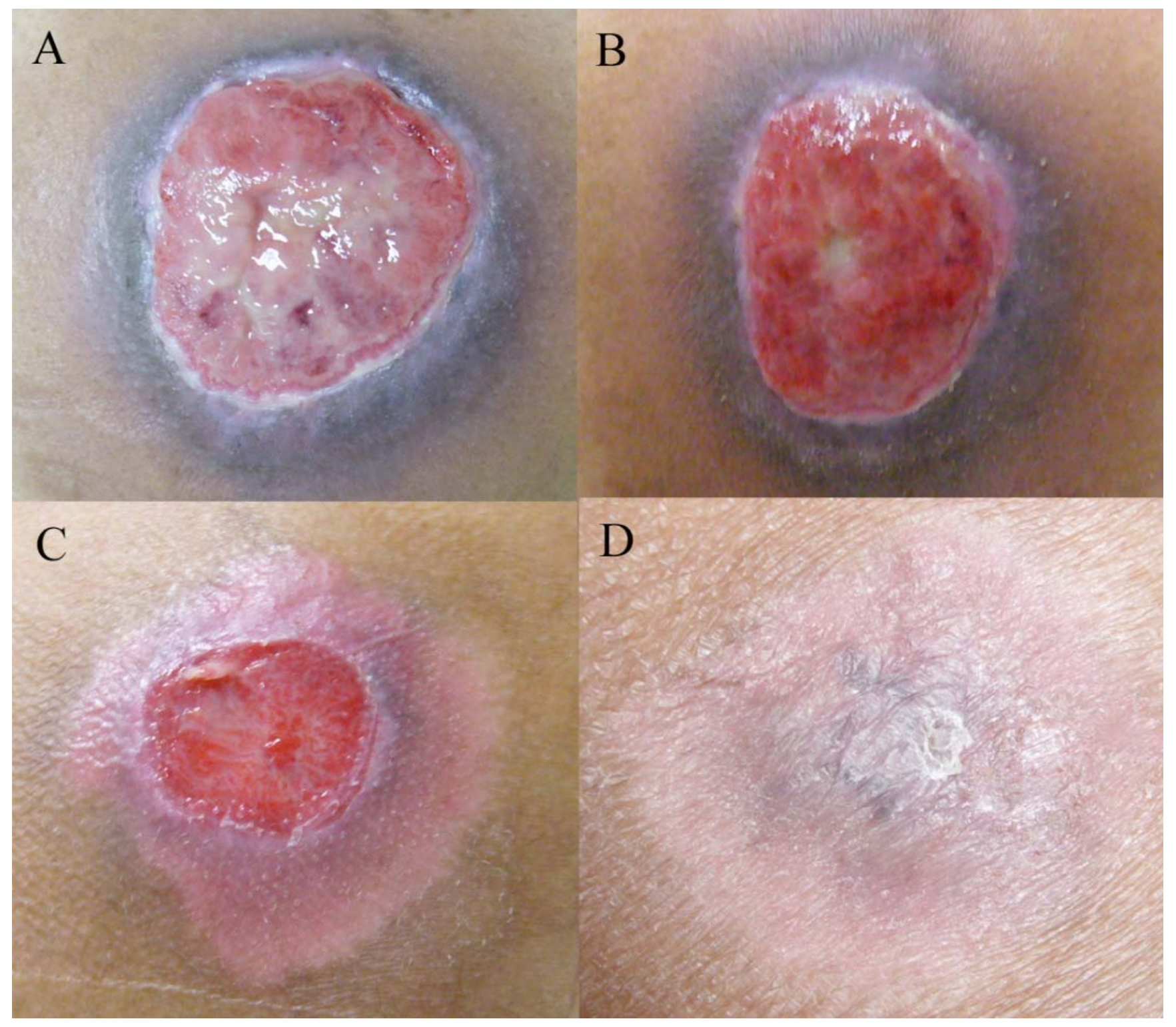

Fig. (1). Clinical course of our patient. A 56-year-old Japanese man developed pressure ulcer on his greater trochanter, which was not improved in spite of applying $0.003 \%$ alprostadil alfadex ointment for two months (A). The $0.9 \%$ cadexomer-iodine ointment was applied once a day for a week. Then, the discharge remarkably decreased (B). After using the cadexomer-iodine ointment for a week, the cadexomeriodine ointment was withdrawn and polyurethane foams were applied. One month later the wound size gradually decreased (C). Applying them further for one month, the wound was completely epithelized (D).

the two signs such as poor healing and exudate were detected to indicate critical colonization but none of the signs for deep infection were shown. Therefore, our patient is retrospectively considered as having pressure ulcer complicated with critical colonization but not deep infection, as these criteria were not available when we treated this patient. For diagnosing critical colonization, the $10^{5}$ bacterial growth guideline [1] is widely appreciated but the patient's severe mental condition prevented us from performing skin biopsy in our patient. In such instances the UPPER and LOWER criteria were useful to evaluate critical colonization. Because the result of swab cultures decreased from the quadrant III to the quadrant I culture using the cadexomer-iodine ointment in our case, this reduction of the bacterial burden due to critical colonization supposedly exerted preferential effect on the healing process.

\section{CONFLICT OF INTEREST}

The authors confirm that this article content has no conflict of interest.

\section{ACKNOWLEDGEMENTS}

Declared none. 


\section{REFERENCES}

[1] Bowler PG. The $10^{5}$ bacterial growth guideline: reassessing its clinical relevance in wound healing. Ostomy Wound Manage 2003; 49: 44-53.

[2] Smith DM. Pressure ulcers in the nursing home. Ann Intern Med 1995; 123: 433-42.
[3] Nicolle LE, Orr P, Duckworth H, et al. Prospective study of decubitus ulcers in two long term care facilities. Can J Infect Control 1994; 9: 35-8.

[4] Woo KY, Coutts PM, Sibbald RG. A randomized controlled trial to evaluate an antimicrobial dressing with silver alginate powder for the management of chronic wounds exhibiting signs of critical colonization. Adv Skin Wound Care 2012; 25: 503-8.

Received: April 23, 2013

Revised: July 2, 2013

Accepted: July 3, 2013

(C) Inui et al.; Licensee Bentham Open.

This is an open access article licensed under the terms of the Creative Commons Attribution Non-Commercial License (http://creativecommons.org/licenses/ by-nc/3.0/) which permits unrestricted, non-commercial use, distribution and reproduction in any medium, provided the work is properly cited. 